\title{
Active Prediction of Heart Disease using Techniques of Hybrid Machine Learning
}

\author{
Jayantkumar A. Rathod, Apoorva R, M. Ramakrishna, Gowthami H R, Rachana T
}

\begin{abstract}
In this world one of the main sources of death is dependent on coronary illness happens in both men and women. It might cause because of the absence of data or inadequate data gave by the doctor in light of some innovation issue or because the prediction level is low. We have additionally observed the utilization of ML methods in ongoing advancements in different Internet of Things (IoT) fields. Different examinations just give a brief look at anticipating coronary illness utilizing ML methods. In this paper, we are looking at how this hybrid method is better than utilizing a single calculation which gives higher exactness up to $88.7 \%$ than contrast with different procedures

Keywords: Algorithms of classification, cardiovascular disease (CVD), Machine learning, Model of prediction, Prediction of heart disease, Selection of functions
\end{abstract}

\section{INTRODUCTION}

The idea of our heart is so flawlessly sorted out and furthermore it is so hard to perceive on account of numerous fundamental hazard factors, for example, diabetes, hypertension, raised cholesterol, sporadic heartbeat cadence and a few different components it is hard to distinguish coronary illness. Various strategies have been utilized in information mining and neural systems to survey the degree of cardiovascular illness among people. It has been characterized in different strategies like Naïve bayes, Decision tree, and SVM and weighted fuzzy principle. In this investigation, different readings were performed to create a prescient model utilizing particular procedures as well as at least two strategies to associate these methodologies together are commonly known as half and half techniques. This strategy utilizes fruitful affiliation rules induced with the GA

Revised Manuscript Received on June 15, 2020.

* Correspondence Author

Jayantkumar A*, Department of Information Science and Engineering, Alva's Institute of Engineering and Technology, Moodbidri, India.

Email: jayantkumarrathod@gmail.com.

Apoorva R, Department of Information Science and Engineering, Alva's Institute of Engineering and Technology, Moodbidri, India.

Email: apoorvarajr@gmail.com.

Gowthami H R, Department of Information Science and Engineering,

Alva's Institute of Engineering and Technology, Moodbidri, India.

Email: gowthamihr2666@gmail.com.

Ramakrishna M, Department of Information Science and Engineering, Alva's Institute of Engineering and Technology, Moodbidri, India.

Email: ramakrishna7748@gmail.com.

Rachana T, Department of Information Science and Engineering, Alva's Institute of Engineering and Technology, Moodbidri, India.

Email: rachanagowda543143@gmail.com.

(C) The Authors. Published by Blue Eyes Intelligence Engineering and Sciences Publication (BEIESP). This is an open access article under the CC BY-NC-ND license (http://creativecommons.org/licenses/by-nc-nd/4.0/) for the choice, hybrid, and transformation of the competition which brings about the new wellness include proposed. We utilize the notable Cleveland dataset, which is accumulated from a UCI AI vault for exploratory approval.

The forecast for coronary illness depends on symptoms, to be specific heartbeat rate, sex, age, and numerous others. Inside this work, we present a strategy with the Linear Model (HRFLM) called the Hybrid Random Forest. The principal objective of this examination is to improve coronary illness expectation yield exactness, there have been a few investigations that bring about capacity choice requirements for algorithmic uses alternately, and the HRFLM approach utilizes all usefulness with no imperatives on the assortment of usefulness. We likewise presented an effective clinical choice decision support system in the presented studies, utilizing fuzzy logic, in which weighted fuzzy principles are utilized, that are created automatically. In this paper, we are going to look at between two strategies that are hybrid AI procedure versus a single AI calculation that is a weighted fuzzy principle.

\section{RELATED WORK}

Many researchers and scholars have focused on prediction of heart disease and classification activities, and have reported their findings. We saw that many of the related works were done using the methodologies of deep learning and machine learning, and were done using single algorithms. In this paper we used the HRFLM to predict modified data set for our research; this HRFLM consists mainly of three algorithms. The 13 UCD-Cleveland dataset is used by SVM.DT and NB to achieve greater accuracy compared to that based on sensitivity, precision and specificity.

\section{INPUT SOURCES:}

In this paper we used 3 main algorithms namely 1). SVM Algorithm 2). Naive Bayes Classifier Algorithm 3). Decision tree Classification.

NAIVE BAYES: It is a method of the order dependent on Bayes's hypothesis, with an assumption of independence between predictors. Basically, a Naive Bayes classifier accepts that the nearness of a particular component in a class isn't identified with some other feature. $\mathrm{P}(\mathrm{C} \backslash \mathrm{X})=\mathrm{P}$ $(\mathrm{X} \backslash \mathrm{C}) * \mathrm{P}(\mathrm{C}) \mathrm{P}(\mathrm{X}) \mathrm{P}(\mathrm{C})=$ =independent likelihood of $\mathrm{C}$ (prior probability) $\mathrm{P}(\mathrm{X})=>$ independent likelihood of $\mathrm{X} \mathrm{P}(\mathrm{X} \backslash \mathrm{C})=>$ conditional likelihood of $\mathrm{X}$ given $\mathrm{C}$ (probability).

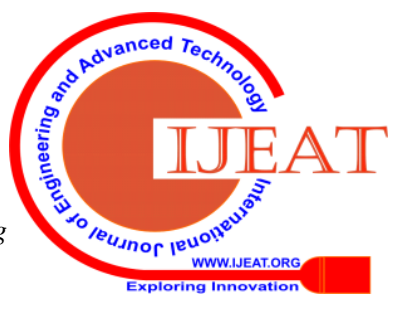




\section{Active Prediction of Heart Disease using Techniques of Hybrid Machine Learning}

DECISION TREE: The decision tree is a regulated calculation for AI. It handles both numerical and downright information. It gives an unmitigated arrangement, for example, Yes/No, True or False, 1 or 0 , contingent upon specific conditions.

\section{Algorithm: Decision Tree-Based Partition}

Input: D dataset - highlights with an objective class for $\forall$ highlights do

For each example do

Execute the Decision Tree calculation

end for

Distinguish the element space $\mathrm{f1}, \mathrm{f} 2$... fx of dataset UCI.

end for

Acquire absolute no. of leaf hubs 11, 12, 13... In with its requirements

Split dataset D into d1, d2... dn dependent on leaf nodes requirements.

Output: datasets for segments $d 1, \mathrm{~d} 2, \mathrm{~d} 3 \ldots \mathrm{dn}$

SUPPORT VECTOR MACHINE: Support Vector Machine or SVM is one of the most well-known Supervised Learning calculations utilized for arrangement just as for issues with relapse. For Machine Learning, be that as it may, it is utilized basically for classification issues.

\section{Algorithms for Classifying Large Datasets}

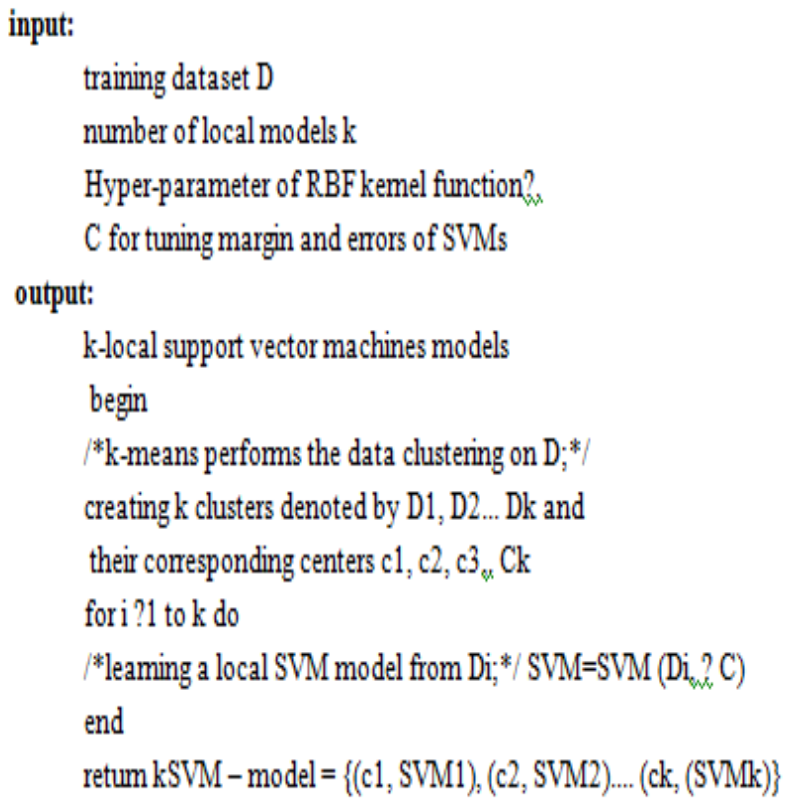

\section{OVERVIEW OF METHOD:}

\subsection{Hybrid Architecture:}

Design of this model says about how to investigate the patient subtleties as a result of the insignificant data in the coronary illness datasets, the first crude information can't be utilized legitimately in the prediction procedure, so crude information should be washed, prepared and changed over for additional means in the pre-handling period of the information. The data accessible focuses on the end that ladies have less possibility of creating coronary illness contrasted with men. The exact conclusion is basic in coronary illness. Fig 1 shows the work process of the UCI dataset.

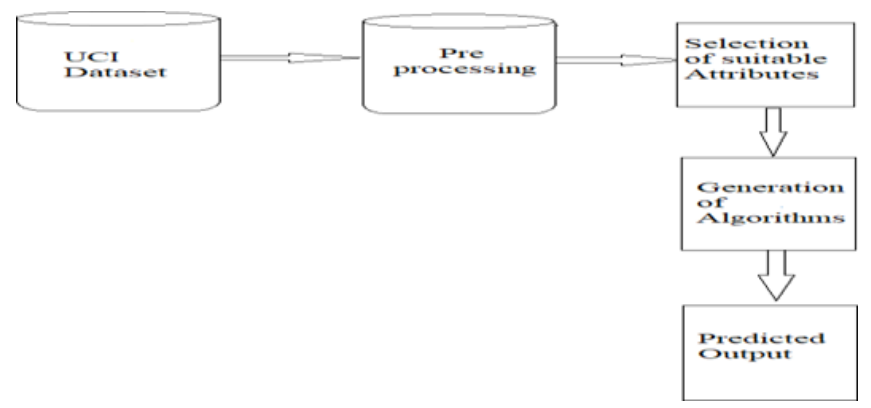

Fig 1. Workflow of UCI dataset

\subsubsection{UCI Dataset:}

The informational dataset is taken from University of California's Data Mining Repository; Irvine (UCI) (Newman et al., 1998).The device is completely checked utilizing the Switzerland, Cleveland, and Hungarand Switzerland dataset. Absolutely 14 attributes-objectives, for example age, sex, circulatory strain resting, kind of chest pain, serum cholesterol in $\mathrm{mg} / \mathrm{dl}$, fasting blood sugar, most extreme pulse came to, angina actuated exercise, rest electro-cardio graphic tests, ST melancholy, top exercise incline ST area, number of significant vessels, and coronary illness determination are given in those datasets.

\section{- Cleveland data}

Such information was acquired by Robert Detrano, M.D., Ph.D., at V.A.Institute for Medicine. All detailed research identifying with the utilization of a subset of 14 of the 76 attributes present in the Cleveland coronary illness database broke down. ML analysts in reality just utilize the Cleveland database until today. In the objective zone, the presence of coronary illness in the patient is shown by methods for a whole number which can take any an incentive from 0 (no nearness) to 4 . Recognizing the nearness of infections (values 1-4) from non-presence (esteem 0 ) was the subject of the led explores in the Cleveland database.

\section{- Hungarian data}

Such information was gotten at the Hungarian Institute of Cardiology, Budapest, by Andras Janosi, M.D. Three of the characteristics have been disposed of because of a colossal level of missing values yet the information structure is actually equivalent to that of the Cleveland results. Thirty-four examples were disposed of because of missing values and there were 261 examples. Class pro-portion is 62.5 percent missing from coronary illness and 37.5 percent present from coronary illness (Bradley, 1997).

\section{- Switzerland data}

This information was gathered by William Steinbrink, M.D., at University Hospital, Zurich, Switzerland. There is increasingly number of missing qualities in Switzer-land. It incorporates 123 cases of information, and 14 attributes. Class extents are missing from 6.5 percent coronary illness and 93.5 percent heart sickness. A detail of these data is shown below as Fig 2.

Published By:

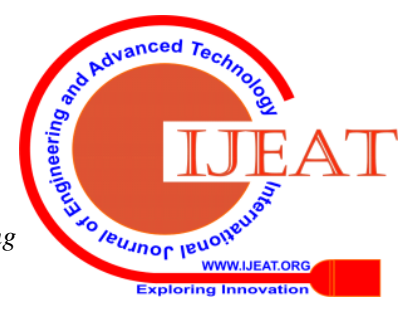




\section{Total instance Training data Testing data}

\begin{tabular}{lrrr}
\hline Cleveland & 303 & 202 & 101 \\
Hungarian & 294 & 196 & 98 \\
Switzerland & 123 & 82 & 41 \\
\hline
\end{tabular}

Fig 2. Details of the Dataset

\subsubsection{Pre-processing:}

The point of preprocessing information is to extricate helpful information from datasets of raw coronary illness and afterward this information ought to be converted into the configuration fitting for risk level forecast. Due to the unessential data in the coronary illness datasets, the first crude information can't be utilized legitimately in the forecast strategy, so crude information should be washed, handled and changed over for additional means in the pre-preparing period of the information. Along these lines, the unimportant attributes are discovered utilizing the procedure examined in choosing fitting properties, and in the wake of killing the irrelevant ones, they are transformed into row-column format.

\subsubsection{Selection of suitable attributes:}

The reason for information pre-processing is to extract helpful information from raw coronary illness datasets and afterward this information ought to be changed over into the organization vital for the expectation of hazard level. Because of the unessential data in the coronary illness datasets, the first raw information can't be legitimately utilized in the forecast technique, subsequently in information pre-processing stage, raw.

\subsubsection{Generation of algorithms:}

The grouping of informational collections is done based on the Decision Tree (DT) highlights factors and parameters. Rather, to estimate its yield, the classificatory are applied to each bunched dataset. From the above outcomes, the best performing models are recorded, in light of their low error rate. By choosing the DT cluster with a high error rate and expelling its comparing classifier includes, the productivity is additionally advanced. The classifier execution is assessed on this informational index for error streamlining.

\subsubsection{Prediction output:}

In this stage the clinical decision support system exploratory outcomes for hazard forecast are explained. Here, to survey the affectability, explicitness and exactness, the exhibition of the proposed framework is contrasted and the neural system based framework.

\subsection{Single Algorithm (Weighted Fizzy Algorithm):}

As individuals have as of late checked out their health, one of the best research zones has been the advancement of clinical area application. One case of the clinical area application is the coronary illness location program dependent on computer based application helped demonstrative techniques, where the information is gotten from different sources and broke down based on computer based applications. Prior, the utilization of computers was to make a data based clinical decision making support system that physically deciphers this data through computer calculations utilizing mastery from clinical specialists. This technique requires significant investment and depends on the perspectives on clinical specialists, which might be abstract. AI methods were created to deal with this issue to naturally pick up information from models or raw information. For the conclusion of coronary illness, a weighted fluffy principle based clinical decisions supportive system (CDSS) is given here, which promptly obtains data from the clinical information of the patient as appeared in the fig 3.

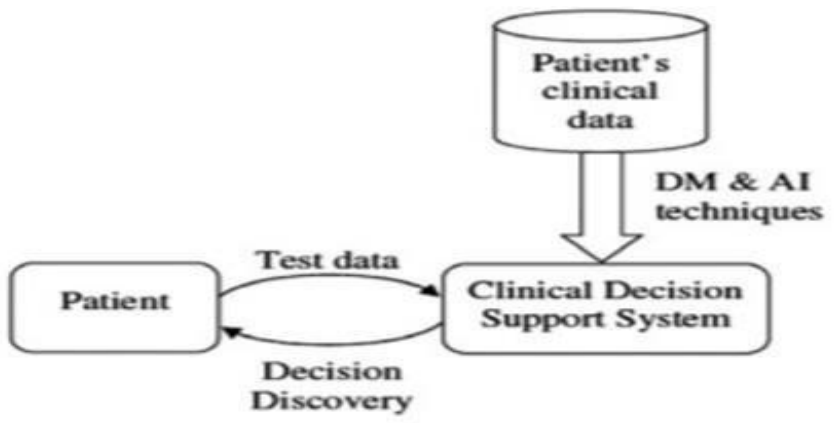

Fig 3. Clinical decision support system

\subsubsection{Data pre-processing:}

The point of preprocessing information is to separate valuable information from datasets of raw coronary illness and afterward these information ought to be converted into the arrangement required for hazard level expectation. On account of the unessential subtleties in the datasets of heart disease, the first crude information can't be utilized straight forwardly in the forecast technique, along these lines, crude information must be cleaned in the preprocessing step, broke down and changed for additional means.

\subsubsection{Classification of training data set:}

The information preparing dataset utilized for expectation is isolated into two subset of many reports handling, in view of the class mark characterized in the information. Actually, coronary illness is commonly an issue with the veins, for instance blocking blood stream or narrowing the vein. There are two execution bunches for coronary illness conclusion, under 50 percent narrowing in distance across (no coronary illness) or in excess of 50 percent narrowing in width (heart disease). Here, value 0 doesn't present the nearness of coronary illness which implies fewer than 50 percent narrowing in measurement and qualities 1-4 show the nearness of coronary illness which implies in excess of 50 percent breadth. We changed the chose UCI information into two class names, appropriately.

\subsubsection{Automated approach to generate weighted fuzzy:}

This segment portrays the robotized approach for creating the weighted fuzzy rule from the ordered dataset to gain proficiency with the fuzzy strategy viably. An enormous number of attributes are given by considering the datasets of coronary illness however the properties of extraction this segment depicts the computerized approach for producing the weighted fuzzy guidelines from the grouped dataset to become familiar with the fuzzy technique. An enormous number of properties are given by considering the datasets of coronary illness yet the attributes of extraction.

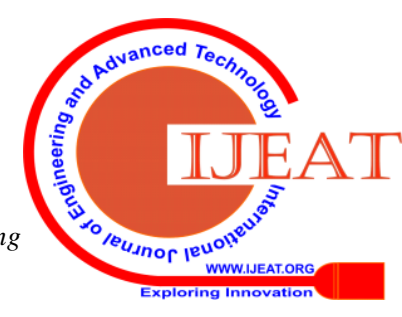




\section{Active Prediction of Heart Disease using Techniques of Hybrid Machine Learning}

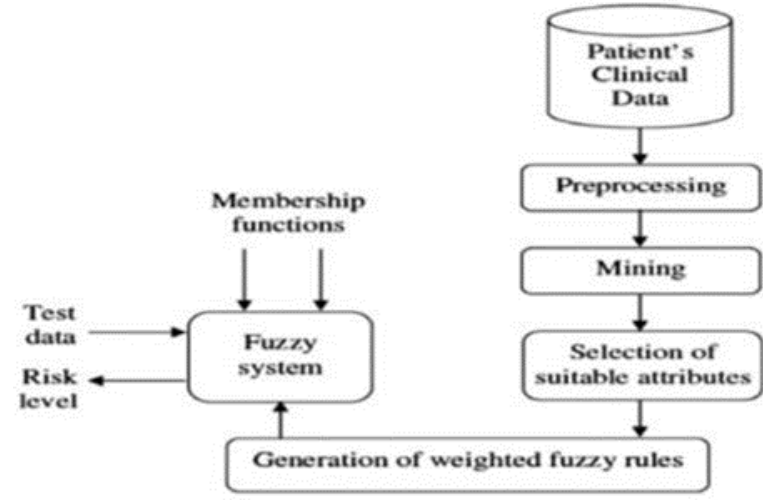

Fig 4. Clinical decision support system based on proposed fuzzy

\subsubsection{Mining of attributes category:}

All through this progression the successive classification of attributes relating to every bi property gave in the $\mathrm{DHj}$ datasets ought to be mined so the recurrence of every class of traits inside the $\mathrm{Cj}$ class is acquired by checking the database. The recurrence of classification of properties is controlled by finding the event of the classification of characteristics in the whole dataset. In order to find the frequency, we discrete it in equi-width for continuous attributes. Here, notable calculations, for example, Apriori (Agrawal et al, 1993) and FP-development (Han et al., 2004) are not appropriate for normal property class mining, as these data formats vary from data suited to conventional algorithms.

\subsubsection{Choosing the appropriate attributes:}

The right qualities are characterized utilizing the two vectors, $\mathrm{Vj}$ max and $\mathrm{Vj}$ Min is acquired from the past stage. The clarification behind this move is that the input information incorporates an enormous number of properties, where all the properties are not so efficient in predicting the risk level of the heart patient. Identifying appropriate characteristics would also ensure greater precision in estimation of the risk level. We have utilized the deviation way to deal with characterize the right traits, where the mined length property type is utilized. The deviations go for the entire thing introduced in the two classes least vector, V1 min and V2 min, is characterized by a coordinated correlation of the particular position.

\subsubsection{Generation of weighted rules:}

The formation of rules and the weighting of rules are a significant advance in creating a Fuzzy based clinical decision support. The deviations vectors, Dmin and Dmax are acquired from the previous step are utilized to generate the rules of decision specifying the degree of risk in terms of numerical variables of heart patients. The rules are generated automatically from the two deviation vectors that comprise the variance of each attribute, compared to two groups.

\subsubsection{Finding weighted fuzzy rule:}

The extremely important task of creating fuzzy rules using numeric symbolic values from the data mentioned appears to be extremely difficult. It is particularly important to treat these types of values as it is so similar to human understanding and laws for these values are generally more comprehensible and accountable compared to the laws on numerical values. Implementing the fuzzy set theory allows this value to be treated which leads to the generation of a collection of fuzzy rules through the construction of fuzzy. The automated method proposed here depends on the development. We accordingly modified the selected UCI data to 2 class labels, accordingly. Automated method for generating weighted fuzzy regulations.

\section{DISCUSSION AND RESULTS:}

\section{Single algorithm:}

This observation deals about the weighted fuzzy rule that says MAT-LAB (7.10) was used to implement the proposed fuzzy, logic-based method of clinical decision support. They took for research the Cleveland, Hungarian and Swiss heart disease dataset, which is commonly, accepted datasets collected from the machine learning repository of UCI. In the testing cycle, the experimental data set is given to the proposed method for assessing the risk prediction of heart patients, and the obtained results are evaluated using the assessment metrics, namely specificity, sensitivity and accuracy (Zhu et ., 2010).

\section{Hybrid algorithm:}

This is about the hybrid algorithm were we can observe $\mathrm{R}$ Studio Rattle further classifies and classes the data set in the proposed model. The results are created by applying data set classification law. The classification rules created after pre-processing of the data are done based on the law. The 3 best ML methods of the data are selected after pre-processing and the outcomes are produced. The various DT, SVM, and NB datasets are implemented to figure out the correct form of classification. The tests demonstrate the best of both DT and SVM. Compared to other datasets, the DT blunder rate for dataset 4 is high (20.9 percent). Contrasted with DT and WFY models, the dataset DT approach is the best (9.1 percent). The DT method is combined with SVM and NB we recommend HRFLM technique to boost the performance.

\section{Benchmarking of the proposed model:}

Benchmarking is expected to think about the exhibition of the Using Weighted Fuzzy Models contrasted with the model proposed. This methodology is utilized to survey if the methodology proposed is the right, and to improve precision or not. The accuracy is determined by the number of selected features and the outcomes produced by the model. HRFLM has no limitations in the scope of functions to be utilized. The entirety of the features selected this model produce the best outcomes. The overall accuracy of the proposed model exceeded $88.7 \%$ and $57.815 \%$ of the Fuzzy system model. Therefore our model accuracy is better similar to the Fuzzy one. The methodology proposed is utilized and evaluated on every one of the 13 characteristics based on the rate of error. This outcome obviously shows that the entirety of the selected features and ML methods utilized are effective in foreseeing patients' coronary illness dependably contrasted with the fuzzy based models.

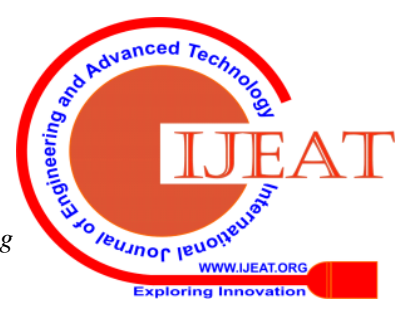




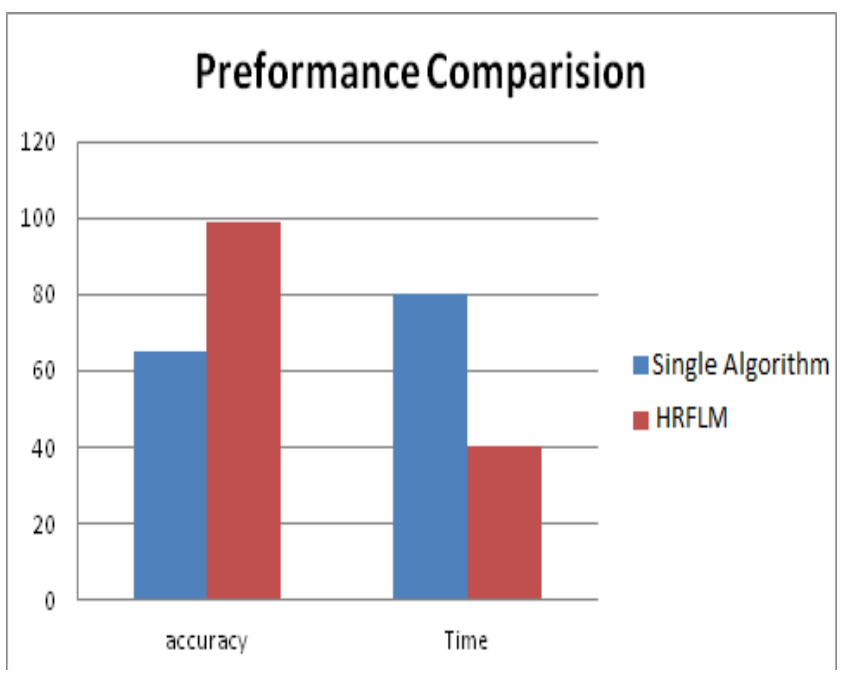

\section{CONCLUSION}

Recognizing the transmission of raw cardiovascular data health information will assist with sparing human lives in the long term, and early discovery of coronary illness abnormalities. Foreseeing coronary illness is troublesome in the clinical division and is significant. Regardless, if the sickness is analyzed in the beginning times and preventive move is made at the earliest opportunity, there could be a significant decrease in mortality chance. AI procedures were utilized to process raw information and to give new and novel wisdom. Here we have made a comparison between single algorithms that's weighted fuzzy versus hybrid algorithm which consist of SVM, DT, NB clearly can observe the results through the graph how the hybrid algorithm stands out in a better platform to predict cardiovascular disease with 88.7 percent accuracy.

\section{REFERENCES}

1. A. S. Abdullah and R. R. Rajalaxmi, "A data mining model for predicting the coronary heart disease using random forest classifier," in Proc. Int. Conf. Recent Trends Comput. Methods, Commun. Controls, Apr. 2012, pp. 22-25.

2. N. Al-milli, "Backpropogation neural network for prediction of heart disease,' J. Theor. Appl.Inf. Technol., vol. 56, no. 1, pp. 131-135, 2013.

3. A. H. Alkeshuosh, M. Z. Moghadam, I. Al Mansoori, and M. Abdar, "Using PSO algorithm for producing best rules in diagnosis of heart disease,' in Proc. Int. Conf. Comput. Appl. (ICCA), Sep. 2017, pp. 306- 311.

4. C. A. Devi, S. P. Rajamhoana, K. Umamaheswari, R. Kiruba, K. Karunya, and R. Deepika, "Analysis of neural networks based heart disease prediction system," in Proc. 11th Int. Conf. Hum. Syst. Interact. (HSI), Gdansk, Poland, Jul. 2018, pp. 233-239.

5. P. K. Anooj, "Clinical decision support system: Risk level prediction of heart disease using weighted fuzzy rules,' J. King Saud Univ.-Comput. Inf. Sci., vol. 24, no. 1, pp. 27-40, Jan. 2012. doi: 10.1016/j.jksuci.2011.09.002.

6. L. Baccour, "Amended fused TOPSIS-VIKOR for classification (ATOVIC) applied to some UCI data sets,'’ Expert Syst. Appl., vol. 99, pp. 115-125, Jun. 2018. doi: 10.1016/j.eswa.2018.01.025.

7. M. Gandhi and S. N. Singh, "Predictions in heart disease using techniques of data mining", Proc. Int. Conf. Futuristic Trends Comput. Anal. Knowl. Manage. (ABLAZE), pp. 520-525, Feb. 2015.

8. C.-A. Cheng and H.-W. Chiu, "An artificial neural network model for the evaluation of carotid artery stenting prognosis using a national-wide database," in Proc. 39th Annu. Int. Conf. IEEE Eng. Med. Biol. Soc. (EMBC), Jul. 2017, pp. 2566-2569.

9. M. Durairaj and V. Revathi, "Prediction of heart disease using back propagation MLP algorithm", Int. J. Sci. Technol. Res., vol. 4, no. 8, pp. 235-239, 2015.

10. H. A. Esfahani and M. Ghazanfari, "Cardiovascular disease detection using a new ensemble classifier,' in Proc. IEEE 4th Int. Conf. Knowl. Based Eng. Innov. (KBEI), Dec. 2017, pp. 1011-1014.

11. F. Dammak, L. Baccour, and A. M. Alimi, "The impact of criterion weights techniques in TOPSIS method of multi-criteria decision making in crisp and intuitionistic fuzzy domains,', in Proc. IEEE Int Conf. Fuzzy Syst. (FUZZ-IEEE), vol. 9, Aug. 2015, pp. 1-8.

12. R. Das, I. Turkoglu, and A. Sengur, "Effective diagnosis of heart disease through neural networks ensembles," Expert Syst. Appl., vol. 36, no. 4, pp. 7675-7680, May 2009. doi: 10.1016/j.eswa.2008.09.013.

\section{AUTHORS PROFILE}
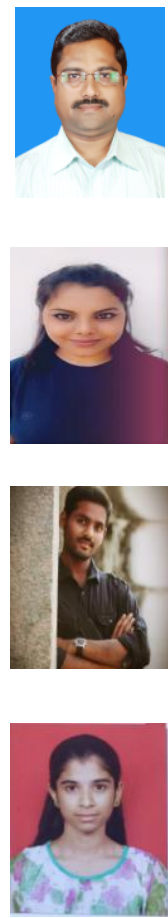

Gowthami H R, Final year B.E, Department of Information Science and Engineering, Alva's Institute of Engineering and Technology, Mijar, Karnataka, India. Email: gowthamihr2666@gmail.com.

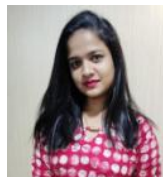

Rachana T, Final year B.E, Department of Information Science and Engineering, Alva's Institute of Engineering and Technology, Mijar, Karnataka, India. Email: rachanagowda543143@gmail.com.

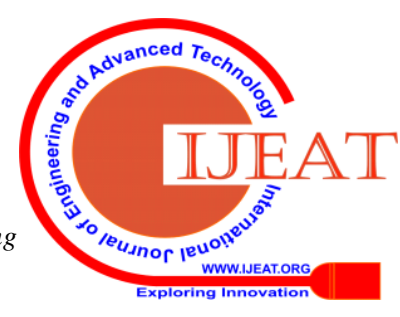

